\title{
LISTADO FlORÍSTICO DE LA SIERRA DE Santa Rosa, Guanajuato, MÉxico
}

\author{
Juan MartíneZ-CruZ1, ${ }^{2}$ Y OSWALdo TÉLlez-Valdés ${ }^{3}$ \\ ${ }^{1}$ Departamento de Biología, Instituto de Biología, Universidad Nacional Autónoma de México. \\ ${ }^{2}$ Dirección actual: Centro de Investigaciones en Ecosistemas, Universidad Nacional Autónoma de México, \\ Antigua Carretera a Pátzcuaro 8701, Col. Ex Hacienda de San José de la Huerta, Morelia 58190, Michoacán, \\ México. Tel. (443) 322-2704, ext. 42508; Correo-e: jmc@oikos.unam.mx \\ ${ }^{3}$ Facultad de Estudios Superiores Iztacala, Unidad de Biología, Tecnología y Prototipos. \\ Universidad Nacional Autónoma de México, Av. de los Barrios 1, Los Reyes Iztacala, Tlalnepantla 54090, \\ Edo. de México, México. Correo-e: tellez@servidor.unam.mx
}

\begin{abstract}
Resumen: Actualmente la Sierra de Santa Rosa es una de las pocas áreas reminiscentes medianamente conservadas en Guanajuato, ya que desafortunadamente el estado se ha caracterizado por la degradación de su cubierta vegetal desde tiempos coloniales. En este estudio florístico se registran 21 taxa infraespecíficos, 496 especies, 273 géneros y 93 familias de plantas vasculares. De éstas, la familia Asteraceae tiene la mayor riqueza de especies, seguida por Poaceae y Lamiaceae. Se registraron tres especies (Selaginella porphyrospora, Zigadenus virescens y Gentiana spathacea) que están incluidas en la Norma Oficial Mexicana de Especies Amenazadas. Se encontró que la riqueza florística de parte de esta sierra es significativa, ya que a pesar de representar una proporción relativamente pequeña (aprox. 0.45\%) del área que abarca el proyecto Flora del Bajío y de Regiones Adyacentes (que ocupa alrededor de 50,000 $\mathrm{km}^{2}$ ), el número de especies equivale a $8.7 \%$ del número estimado para esa flora.
\end{abstract}

Palabras clave: bosque de Quercus, florística, Guanajuato, México, Sierra de Santa Rosa.

Abstract: The Santa Rosa range presently contains one of the few moderately preserved vegetation remnants in the state of Guanajuato. Unfortunately, this state has been characterized by the degradation of its vegetation cover since colonial times. In this floristic study we recorded 21 infraspecific taxa, 496 species, 273 genera and 93 families of vascular plants. Among these, Asteraceae comprises the largest number of species, followed by Poaceae and Lamiaceae, respectively. Three species cited in the Mexican Norm of Endangered Species (Selaginella porphyrospora, Zigadenus virescens and Gentiana spathacea) were recorded. Despite the reduced area of this mountain range, which represents around $0.45 \%$ of the area covered by the Flora of the Bajío and of Adjacent Regions project (about 50,000 $\mathrm{km}^{2}$ ), its floristic richness represents $8.7 \%$ of the total flora expected for that region.

Key words: Quercus forest, floristics, Guanajuato, Mexico, Santa Rosa range.

$\mathbf{L}$ a flora mexicana está reconocida entre las más diversas de la tierra, ya que se ha estimado que posee entre 22,350 y 25,000 especies (Rzedowski y Equihua, 1987; Rzedowski, 1991; Villaseñor, 2003). Asimismo, su endemismo es notable, pues $10 \%$ de los géneros y $52 \%$ de las especies son exclusivas del país. Sin embargo, a pesar de que actualmente numerosas floras regionales abarcan y brindan el conocimiento florístico de casi tres cuartas partes del país, se estima que $30 \%$ de su superficie no ha sido cubierta aún por los estudios florísticos, y que existen áreas insuficientemente exploradas. Éste es el caso de los estados de Zacatecas, Colima, Campeche y Guanajuato, para los cuales no existen prácticamente listados florísticos (Dávila y Sosa, 1994).

De acuerdo con lo anterior, Rzedowski (1997) señala que en la zona del Bajío y regiones adyacentes (Guanajuato, Querétaro y el norte de Michoacán), con un área aproximada de 50,000 $\mathrm{km}^{2}$, existen alrededor de 5,500 especies de plantas vasculares. Por lo anterior, se consideró apropiado realizar un estudio de la flora de una porción de la Sierra de Santa Rosa, Guanajuato, con el objetivo de elaborar un listado florístico preliminar.

Las colecciones botánicas en la región del Bajío y la Nueva Galicia se remontan a finales del siglo XVIII por 
José Mariano Mociño y Luis Née. Posteriormente, la recolección de ejemplares se incrementó en forma significativa durante el primer cuarto del siglo XIX, aunque fue realizada en forma irregular y eventual por diversos colectores, como L. Alamán, A.J.A.G. Bonpland, A. Dugès, K.T. Hartweg y J.N. Rose, cuyas colecciones se encuentran depositadas primordialmente en herbarios de Estados Unidos y Europa (McVaugh, 1972).

Durante el siglo pasado se efectuó el mayor esfuerzo para lograr un conocimiento florístico del centro y occidente del país, aunque sin dar énfasis al estado de Guanajuato, sino considerándolo como parte de los estudios florísticos del Bajío y de la Nueva Galicia. El primero en elaborar un listado sustancial fue Dugès (1924). Posteriormente existieron diversos esfuerzos aislados, como los realizados por Aguilera (1991) con su estudio en el cráter del Rincón de Parangueo, o como los de Meyrán (1966) y Rubio (1993), quienes hicieron importantes aportaciones a la florística de Guanajuato. Se cuenta con trabajos en donde solamente se describen algunas especies nuevas como el de Pérez-Calix (1997) o como el de Galván et al. (1994), quienes presentan listados florísticos parciales, o como los de Quero (1977) y Pineda (1978), quienes hicieron aportaciones a la florística del estado con sus trabajos enfocados al estudio de la vegetación forestal del estado.

Tomando en consideración el período en el que se ha realizado mayor esfuerzo para lograr un conocimiento florístico, sólo dos estudios incluyen en sus objetivos, ya sea de forma parcial o total, a la Flora de Guanajuato. Estos son los proyectos "Flora del Bajío y de regiones adyacentes", del cual a la fecha se han publicado numerosos tratamientos de la flora (Rzedowski y Calderón de Rzedowski, 1985; Argüelles et al., 1991; Rzedowski et al., 1991; Díaz y Palacios, 1992; Rzedowski et al., 1995, 1996; Ballard, 1997; Calderón de Rzedowski, 1997a, b, c; Carranza, 1997a, b; Carranza y Madrigal, 1997; Fernández, 1997; Fryxell, 1997; García y Koch, 1997; Graham, 1997; Rodríguez, 1997; Rzedowski, 1997; Rzedowski y Calderón de Rzedowski, 1997a, b, c, d, e, f, g) y la Flora NovoGaliciana (McVaugh y Rzedowski, 1966; McVaugh, 1974, 1983, 1984, 1987a, b, 1989, 1992), proyectos que hasta el momento han logrado un avance sustancial en el conocimiento de la Flora de Guanajuato. Así, es evidente que el conocimiento florístico en particular de la flora de la Sierra de Santa Rosa es muy superficial y fragmentario, y que se encuentra registrado en forma aislada en los diversos trabajos taxonómico-florísticos citados previamente.

\section{Área de estudio}

La Sierra de Santa Rosa se localiza al norte de la ciudad de Guanajuato (figura 1), entre $\operatorname{los} 20^{\circ} 45^{\prime}$ y $21^{\circ} 25^{\prime}$ de latitud

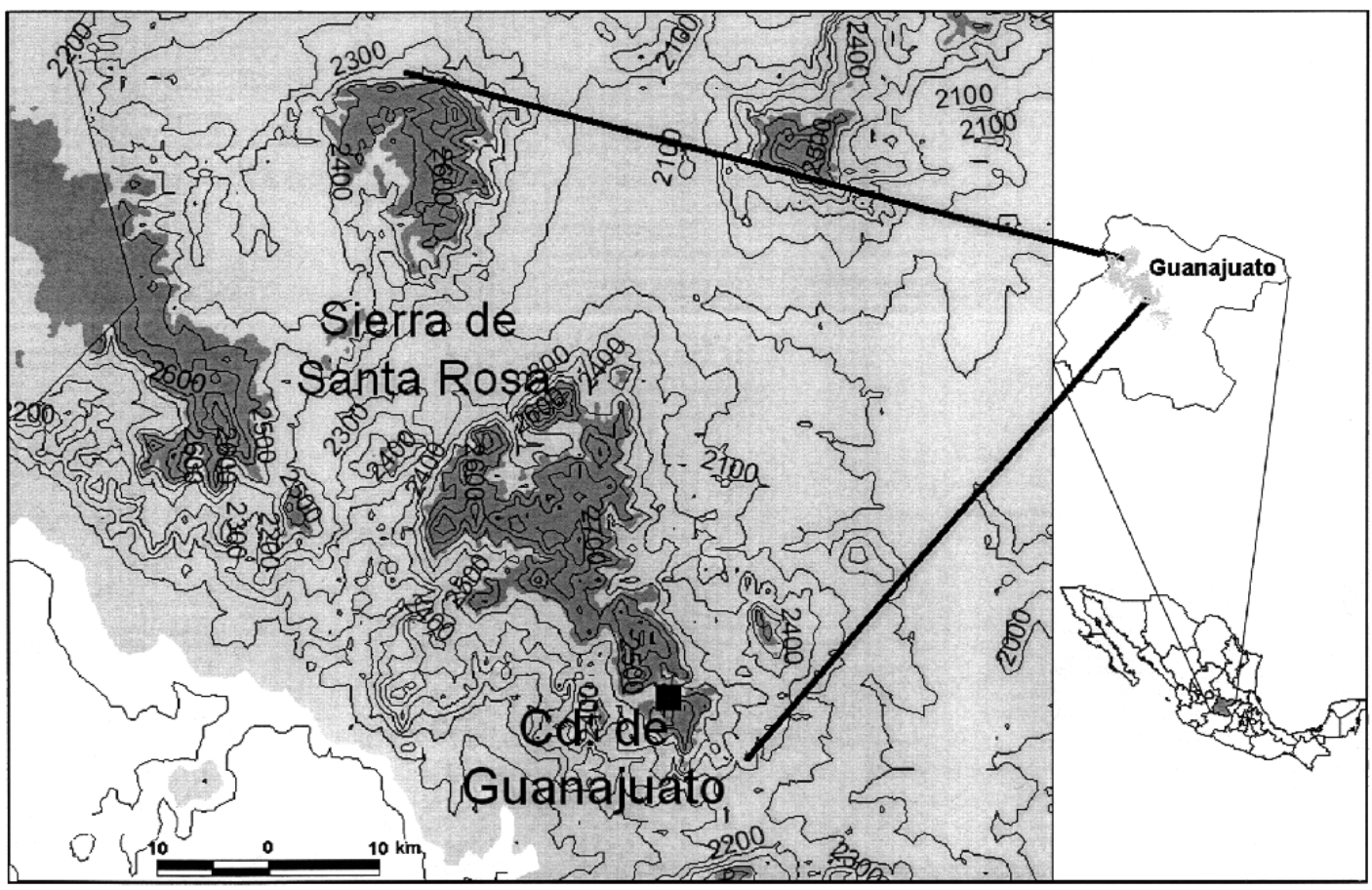

Figura 1. Ubicación de la Sierra de Santa Rosa en el estado de Guanajuato. 
norte y entre los $100^{\circ} 53^{\prime}$ y $101^{\circ} 25^{\prime}$ de longitud oeste. Abarca parte de los municipios de Guanajuato, Dolores Hidalgo y San Felipe. Está considerada dentro de la subprovincia de Sierras y Llanuras del Norte de Guanajuato, en la región fisiográfica de la Mesa Central, que presenta montañas fuertemente disectadas, barrancas y cañadas, con altitudes que oscilan entre 1,800 y 2,700 m. Su geología está conformada por rocas sedimentarias de materiales detríticos y rocas ígneas en forma de basaltos, ignimbritas, riolitas, andesitas y tobas, que datan del Cuaternario (Cuanalo de la Cerda et al., 1989; Anónimo, 1991). Los suelos que ocupan una mayor extensión son los Castañozem (> 60\%), seguidos de Litosoles (25\%) y Rendzina (8\%). Su clima es el de mayor humedad de los templados subhúmedos, con alrededor de $800 \mathrm{~mm}$ de precipitación promedio anual (García, 1981).

En cuanto a la vegetación, se señala principalmente la presencia del bosque de Quercus, mientras que para la vertiente oriental de la sierra se presenta el matorral crasicaule, además de las comunidades vegetales de mezquital y bosque de Pinus-Quercus, cuya distribución se encuentra determinada por las diferentes condiciones ambientales (Anónimo, 1981).

\section{Materiales y métodos}

La recolección botánica se realizó sistemáticamente entre julio de 1997 y agosto de 1998. Se recolectaron tres réplicas en promedio; juegos completos de estas colecciones se encuentran depositados en el Herbario Nacional del Instituto de Biología (MEXU) y en el Herbario del Instituto de Ecología A.C., Centro Regional del Bajío (IEB), mientras que un juego parcial fue depositado en el Herbario de la Facultad de Estudios Superiores Iztacala (IZTA). Con estas colecciones se elaboró un listado florístico en donde las familias, los géneros y las especies se encuentran orde-

Cuadro 1. Número de géneros y especies en las seis familias más ricas, y las familias restantes, y los porcentajes que estos taxa representan con relación al total de la flora de la Sierra de Santa Rosa.

\begin{tabular}{lcccc}
\hline Familias & Géneros & $\%$ & Especies & $\%$ \\
\hline Asteraceae & 46 & 16.6 & 104 & 20.9 \\
Poaceae & 19 & 6.8 & 34 & 6.8 \\
Fabaceae & 17 & 6.2 & 27 & 5.5 \\
Lamiaceae & 8 & 2.9 & 20 & 4.1 \\
Scrophulariaceae & 7 & 2.5 & 18 & 3.6 \\
Fagaceae & 1 & 0.4 & 14 & 2.8 \\
6 familias más ricas & 98 & 35.4 & 217 & 43.7 \\
87 familias restantes & 175 & 64.6 & 279 & 56.3 \\
Total & 273 & 100 & 496 & 100 \\
\hline
\end{tabular}

nados alfabéticamente; sin embargo, se siguen los sistemas propuestos por Cronquist (1981) para las dicotiledóneas, el de Dahlgren et al. (1985) para las monocotiledóneas y el de Mickel et al. (1990) para las pteridofitas. Los nombres de los autores están citados de acuerdo con Brummit y Powell (1992).

Finalmente, una fuente de información importante que permitió enriquecer el presente listado fue la base de datos florística del Herbario Regional del Bajío, Instituto de Ecología A.C., de donde se extrajeron e incorporaron numerosos registros al mismo.

\section{Resultados y discusión}

Se recolectaron 979 números en total, a partir de los cuales se elaboró un listado florístico que incluye un total de 14 variedades, 7 subespecies, 496 especies y 273 géneros de 93 familias de plantas vasculares (ver apéndice 1). Las angiospermas están representadas por 69 especies de monocotiledóneas, así como por 390 especies más y 20 taxa infraespecíficos de dicotiledóneas; mientras que de gimnospermas sólo se registró una especie. Asimismo, se determinaron 36 especies y una variedad de pteridofitas. En el cuadro 1 se presentan los datos de riqueza de las familias más diversas.

La incorporación de los registros obtenidos de la base de datos del herbario IEB incrementó y enriqueció sustancialmente el listado, el cual originalmente estaba basado en los registros obtenidos por medio de la recolección en campo, y que dieron origen a esta publicación. Específicamente, la aportación es de 12 familias, 84 géneros, 216 especies y 10 taxa infraespecíficos; estos registros en general representan un $30-50 \%$ de incremento en géneros y especies, respectivamente, a los registrados por medio del trabajo en campo.

Aunque los números de familias, géneros y especies registrados aquí son cuantiosos, un análisis somero del número de especies de algunas familias en particular, como por ejemplo Fabaceae, Poaceae, Malvaceae, Euphorbiaceae, Cyperaceae y Orchidaceae, revela una riqueza inferior para la Sierra de Santa Rosa que la usualmente reportada en estas familias para tipos de bosques semejantes en otras regiones de México (Rzedowski, 1998). Pensamos que esta riqueza registrada no es real, sino que más bien se debe a falta de exploración para lograr un inventario más completo, que refleje una riqueza más cercana a la comúnmente reportada en este tipo de ambiente.

Por lo anterior, creemos que las estimaciones sobre la riqueza florística de la Sierra de Santa Rosa y en general de la flora del Bajío y regiones adyacentes son conservadoras (Rzedowski 1997), y que pudieran verse superadas por los registros obtenidos en estudios como el presente. En apoyo a esta idea, en el área de estudio se registraron 496 especies, que equivaldrían a $9 \%$ del total calculado por Rzedowski para la Flora del Bajío, en un área cercana a los 
$225 \mathrm{~km}^{2}$, es decir, apenas el $0.45 \%$ de la extensión del área cubierta por el proyecto del Bajío y de regiones adyacentes. Por ello, la riqueza específica registrada para esta porción estudiada relativamente pequeña de la Sierra de Santa Rosa permite inferir que existe una mayor riqueza y diversidad en la misma.

Entre las aportaciones de este estudio está la ampliación de las áreas de distribución geográfica de diversas especies en el estado, las que recientemente han sido reportadas como nuevos registros para la zona nororiental de Guanajuato (Rzedowski et al., 1996). Entre estas especies están Asclepias contrayerba, Cerdia congestiflora, Paronychia mexicana, Conyza gnaphalioides, Dyssodia tenuifolia, Perymenium buphthalmoides, Ipomoea orizabensis, Erysimum capitatum, Lepechinia caulescens, Phaseolus pedicellatus, Malaxis brachystachya, Galium aschenbornii y Rhodosciadium montanum.

Asimismo, es importante señalar que algunas especies comunes y/o de amplia distribución fueron registradas de escasas localidades y colecciones en la Sierra de Santa Rosa. Su escasez parece deberse a la disminución de sus poblaciones y la reducción de sus áreas de distribución, debido al importante impacto causado por la extracción de madera y la producción de carbón. Entre éstas tenemos a Botrychium virginianum, Comarostaphylis polifolia, Chimaphila umbellata, Berberis moranensis, Salix bonplandiana, Galium mexicanum, Thalictrum gibbosum, Ceanothus coeruleus, Ribes affine, Viola guatemalensis y Gentianopsis detonsa ssp. lanceolata. A su vez, este proceso ha originado que especies como Euphorbia dentata, Asphodelus fistulosus, Ageratina glabrata, Stevia salicifolia, Arctostaphylos pungens, Salvia mexicana, Helianthemum glomeratum y Loeselia mexicana se desarrollen en forma masiva, en donde existe una mayor degradación de la capa arbórea, comportándose en la mayoría de los casos como invasoras. También es necesario aclarar que a pesar de que algunas de las especies antes señaladas, entre muchas otras, pueden utilizarse como medicinales, forrajeras o alimenticias, no existe un uso generalizado entre los pobladores de la zona, por lo que el potencial de aprovechamiento de la flora del lugar es muy amplio.

Un rubro de suma importancia a señalar es la presencia de especies consideradas en la Norma Oficial Mexicana de Especies Amenazadas NOM-059 (Diario Oficial de la Federación, 2000), en la zona de estudio. Entre estos taxa encontramos a Selaginella porphyrospora que se encuentra en peligro de extinción, y Gentiana spathacea y Zigadenus virescens, ambas catalogadas como raras. Sin embargo, es importante señalar que Gentiana spathacea es una especie común en distintas partes del país (Rzedowski y Calderón de Rzedowski, 1985), por lo que debe ser revalorada para estar adecuadamente considerada en la Norma Oficial.

Finalmente, a pesar de que la Sierra de Santa Rosa cubre un área relativamente pequeña, esta zona alberga una importante diversidad florística aún no adecuadamente muestreada. Esta conclusión muestra la necesidad de intensificar los estudios florísticos en áreas poco exploradas, o que aparentemente están muy deterioradas, pero que aún conservan una razonable representación de la flora local que merece ser considerada para su adecuada protección.

\section{Agradecimientos}

Queremos agradecer a Angélica Estrada por su gestión de una beca del Consejo Internacional para la Preservación de las Aves en México, institución a la que también se agradece, con el fin de realizar el presente estudio. También agradecemos a diversos especialistas que ayudaron en la determinación de las colecciones: José Luis Villaseñor Ríos (Asteraceae), Isidro Méndez Larios (Scrophulariaceae), Guadalupe Segura Hernández (Verbenaceae), Salvador Arias (Cactaceae), Jorge Sánchez Ken (Poaceae). Al Sr. Miguel Cano por su hospitalidad y ayuda en la recolección botánica. A los curadores Mario Sousa Sánchez (MEXU) y Jerzy Rzedowski (IEB) por las facilidades para la consulta de las colecciones. Asimismo al Dr. Rzedowski, quien sugirió y permitió incorporar a este listado numerosas colecciones y nombres de especies de la base de datos del Instituto de Ecología en Pátzcuaro, con lo cual este listado se enriqueció y actualizó notablemente. Finalmente, a los Dres. Mahinda Martínez y Díaz de Salas y Sergio Zamudio por la revisión crítica del manuscrito y sus valiosas correcciones.

\section{Literatura citada}

Aguilera G.L.I. 1991. Estudio florístico y sinecológico de la vegetación del cráter "Olla de Rincón de Parangueo", Valle de Santiago, Guanajuato. Tesis de Maestría, Colegio de Postgraduados, Montecillos, Estado de México, 99 pp.

Argüelles E., Fernández R. y Zamudio S. 1991. Listado Florístico Preliminar del Estado de Querétaro. Flora del Bajío y de Regiones Adyacentes. Fascículo Complementario II. Instituto de Ecología, A.C., Pátzcuaro, Mich.

Anónimo. 1981. Síntesis Geográfica del Estado de Guanajuato. Coordinación General de Estadística, Geografía e Informática, Guanajuato, Gto.

Anónimo. 1991. Datos Básicos de la Geografía de México. Instituto Nacional de Estadística, Geografía e Informática. 2a ed., Aguascalientes, Ags.

Ballard E.H. 1997. Familia Violaceae. Flora del Bajío y de Regiones Adyacentes. Fascículo 31. Instituto de Ecología, A.C., Pátzcuaro, Mich.

Brumitt R.K. y Powell C.E. Eds. 1992. Authors of Plant Names. Royal Botanic Gardens, Kew, 732 pp.

Calderón de Rzedowski G. 1997a. Familia Cistaceae. Flora del Bajío y de Regiones Adyacentes. Fascículo 2. Instituto de Ecología, A.C., Pátzcuaro, Mich.

Calderón de Rzedowski G. 1997b. Familia Compositae: tribu Lactucea. Flora del Bajío y de Regiones Adyacentes. Fascículo 
54. Instituto de Ecología, A.C., Pátzcuaro, Mich.

Calderón de Rzedowski G. 1997c. Familia Loasaceae. Flora del Bajío y de Regiones Adyacentes. Fascículo 7. Instituto de Ecología, A.C., Pátzcuaro, Mich.

Carranza E. 1997a. Familia Salicaceae. Flora del Bajío y de Regiones Adyacentes. Fascículo 37. Instituto de Ecología, A.C., Pátzcuaro, Mich.

Carranza E. 1997b. Familia Garryaceae. Flora del Bajío y de Regiones adyacentes. Fascículo 49. Instituto de Ecología, A.C., Pátzcuaro, Mich.

Carranza E. y Madrigal X. 1997. Familia Betulaceae. Flora del Bajío y de Regiones Adyacentes. Fascículo 39. Instituto de Ecología, A.C., Pátzcuaro, Mich.

Cronquist A. 1981. An Integrated System of Classification of Flowering Plants. Columbia University Press, Nueva York.

Cuanalo de la Cerda H., Ojeda E., Santos O.A. y Ortiz C.A. 1989. Provincias, Regiones y Subregiones Terrestres de México. Colegio de Postgraduados, Chapingo, Edo. de México.

Dahlgren R.M.T., Clifford H.T. y Yeo P.F. 1985. The Families of the Monocotyledons. Springer-Verlag, Nueva York.

Dávila P. y Sosa V. 1994. El conocimiento florístico de México. Boletín de la Sociedad Botánica de México 55:21-27.

Díaz H. y Palacios M. 1992. Listado Preliminar de las Especies de Pteridofitas de los Estados de Guanajuato, Michoacán y Querétaro. Flora del Bajio y de Regiones Adyacentes. Fascículo Complementario III. Instituto de Ecología, A.C., Pátzcuaro, Mich.

Diario Oficial de la Federación. 2000. Proyecto de Norma Oficial Mexicana PROY-NOM-059-ECOL-2000, Protección ambiental - Especies de flora y fauna silvestres de México - Categorías de riesgo y especificaciones para su inclusión, exclusión o cambio - Lista de especies en riesgo. Secretaría del Medio Ambiente, Recursos Naturales y Pesca. México, D.F.

Dugès A. 1924. Flora y Fauna del Estado de Guanajuato. Imprenta del Estado, Guanajuato, Gto.

Fernández N.R. 1997. Familia Rhamnaceae. Flora del Bajío y de Regiones adyacentes. Fascículo 43. Instituto de Ecología, A.C., Pátzcuaro, Mich.

Fryxell P.A. 1997. Familia Malvaceae. Flora del Bajío y de Regiones Adyacentes. Fascículo 16. Instituto de Ecología, A.C., Pátzcuaro, Mich.

Galván R., Barrios M.A. y Meyrán J. 1994. Plantas suculentas del estado de Guanajuato. Cactáceas Suculentas de México 39:13-17.

García E. 1981. Modificaciones al Sistema de Clasificación Climática de Koeppen. Instituto de Geografía. Universidad Nacional Autónoma de México, México, D.F.

García E. y Koch S.D. 1997. Familia Compositae, Tribu: Cardueae. Flora del Bajío y de Regiones Adyacentes. Fascículo 32. Instituto de Ecología, A.C., Pátzcuaro, Mich.

Graham A.S. 1997. Familia Lythraceae. Flora del Bajío y de Regiones Adyacentes. Fascículo 24. Instituto de Ecología, A.C., Pátzcuaro, Mich.

McVaugh R. 1972. Botanical exploration in Nueva Galicia, Mexico. From 1790 to the present time. Contributions of University of Michigan Herbarium 9:205-357.

McVaugh R. 1974. Fagaceae. Contributions of University of Michigan Herbarium 12:1-93.

McVaugh R. 1983. Gramineae. Flora Novo-Galiciana. A Descriptive Account of the Vascular Plants of Western Mexico.
Vol. 14, The University of Michigan Press, Ann Arbor.

McVaugh R. 1984. Compositae. Flora Novo-Galiciana. A Descriptive Account of the Vascular Plants of Western Mexico. Vol. 12, The University of Michigan Press, Ann Arbor.

McVaugh R. 1987a. Leguminosae. Flora Novo-Galiciana. A Descriptive Account of the Vascular Plants of Western Mexico. Vol. 5, The University of Michigan Press, Ann Arbor.

McVaugh R. 1987b. Orchidaceae. Flora Novo-Galiciana. A Descriptive Account of the Vascular Plants of Western Mexico. Vol. 16, The University of Michigan Press, Ann Arbor.

McVaugh R. 1989. Bromeliaceae-Dioscoreaceae. Flora NovoGaliciana. A Descriptive Account of the Vascular Plants of Western Mexico. Vol. 15, The University of Michigan Press, Ann Arbor.

McVaugh R. 1992. Gymnosperms-Pteridophytes. Flora NovoGaliciana. A Descriptive Account of the Vascular Plants of Western Mexico. Vol. 17, The University of Michigan Press, Ann Arbor.

McVaugh R. y Rzedowski J. 1966. La vegetación de Nueva Galicia. Contributions of University of Michigan Herbarium 9:1-123.

Meyrán J. 1966. Exploración preliminar de una zona del estado de Guanajuato. Cactáceas y Suculentas de México 11:88-93.

Mickel J.T., Wagner Jr. W.H. y Gifford E.M. 1990. Ferns and other lower vascular plants. Encyclopaedia Britannica 19:163-178.

Pérez-Calix E. 1997. Una especie nueva de Echeveria (Crassulaceae) del estado de Guanajuato (México). Acta Botanica Mexicana 38:9-12.

Pineda R.A. 1978. La vegetación forestal del estado de Guanajuato. Bosques y Fauna 1:31-41.

Quero R.H. 1977. La vegetación de las serranías de la cuenca alta del río de la Laja, Guanajuato. Anales del Insituto de Biología de la Universidad Nacinal Autónoma de México, Serie Botánica 47:73-99.

Rodríguez C. 1997. Familia Guttiferae. Flora del Bajío y de Regiones Adyacentes. Fascículo 45. Instituto de Ecología, A.C., Pátzcuaro, Mich.

Rubio C.A. 1993. Contribución al estudio florístico de la Sierra de Los Agustinos, Guanajuato. Tesis Licenciatura. Escuela de Biología, Universidad Michoacana de San Nicolás de Hidalgo, Morelia, Mich. 87 pp.

Rzedowski J. 1991. Diversidad y orígenes de la flora fanerogámica de México. Acta Botanica Mexicana 14:3-21.

Rzedowski J. 1997. Los Principales Colectores Botánicos de Guanajuato, Querétaro y Norte de Michoacán. Flora del Bajio $y$ de Regiones Adyacentes. Fascículo Complementario XVII. Instituto de Ecología, A.C., Pátzcuaro, Mich.

Rzedowski J. 1998. Diversidad y orígenes de la flora fanerogámica de México. En: Ramamoorthy T.P., Bye R., Lot A. y Fa J. Eds. Diversidad Biológica de México, pp. 129-145, Instituto de Biología, Universidad Nacional Autónoma de México, México, D.F.

Rzedowski J. y Equihua M. 1987. Atlas Cultural de México (Flora). Grupo Editorial Planeta, México, D.F.

Rzedowski J. y Calderón de Rzedowski G. 1985. Flora Fanerogámica del Valle de México. Vol. II. Instituto de Ecología, México, D.F.

Rzedowski J. y Calderón de Rzedowski G. 1997a. Familia Bignoniaceae. Flora del Bajio y de Regiones Adyacentes. 
Fascículo 22. Instituto de Ecología, A.C., Pátzcuaro, Mich.

Rzedowski J. y Calderón de Rzedowski G. 1997b. Familia Campanulaceae. Flora del Bajío y de Regiones Adyacentes. Fascículo 58. Instituto de Ecología, A.C., Pátzcuaro, Mich.

Rzedowski J. y Calderón de Rzedowski G. 1997c. Familia Compositae: tribu Anthemideae. Flora del Bajío y de Regiones Adyacentes. Fascículo 60. Instituto de Ecología, A.C., Pátzcuaro, Mich.

Rzedowski J. y Calderón de Rzedowski G. 1997d. Familia Geraniaceae. Flora del Bajío y de Regiones Adyacentes. Fascículo 40. Instituto de Ecología, A.C., Pátzcuaro, Mich.

Rzedowski J. y Calderón de Rzedowski G. 1997e. Familia Leguminosae, Subfamilia Caesalpinoideae. Flora del Bajío y de Regiones Adyacentes. Fascículo 51. Instituto de Ecología, A.C., Pátzcuaro, Mich.

Rzedowski J. y Calderón de Rzedowski G. 1997f. Familia Linaceae. Flora del Bajío y de Regiones Adyacentes. Fascículo 6. Instituto de Ecología, A.C., Pátzcuaro, Mich.

Rzedowski J. y Calderón de Rzedowski G. 1997g. Familia
Polemoniaceae. Flora del Bajío y de Regiones Adyacentes Fascículo 33. Instituto de Ecología, A.C., Pátzcuaro, Mich.

Rzedowski J., Calderón de Rzedowski G. y Galván R. 1991. Presentación, Guía para los Autores y Normas Editoriales. Flora del Bajío y de Regiones Adyacentes. Fascículo Complementario I. Instituto de Ecología, A.C., Pátzcuaro, Mich.

Rzedowski J., Calderón de Rzedowski G. y Galván R. 1995. Los Pastizales Calcífilos del Estado de Guanajuato. Flora del Bajío y de Regiones Adyacentes. Fascículo Complementario IX. Instituto de Ecología, A.C., Pátzcuaro, Mich.

Rzedowski J., Calderón de Rzedowski G. y Galván R. 1996. Nota sobre la Vegetación y la Flora del Noreste del Estado de Guanajuato. Flora del Bajio y de Regiones Adyacentes. Fascículo Complementario XIV. Instituto de Ecología, A.C., Pátzcuaro, Mich.

Villaseñor R.J.L. 2003. Diversidad y distribución de las Magnoliophyta de México. Interciencia 28: 160-167.

Fecha de recepción: 30 de octubre de 2003

Versión corregida: 5 de marzo de 2004

Aceptado: 12 de marzo de 2004 
LiSTADO FLORÍ́STICO DE LA SIERRA DE SANTA ROSA

Apéndice 1. Listado florístico de la Sierra de Santa Rosa, Guanajuato. Las claves de los colectores se muestran a continuación: A.R. López (AR), E. Carranza (EC), E. Pérez (EP), E. Ventura (EV), S. Reyes G. (GR), H. Díaz-Barriga (HD), J. Cano (JC), J. Ceja (JJ), J.D. Galván (JD), J. Martínez (JM), J.R. Pérez (JP), J. Rzedowski (JR), J. Sánchez (JS), L.M. González (LM), M. Cano (MC), R. Aguirre (RA), R. Galván (RG), R. Gutiérrez (RZ), R. Santillán (RS), R. Spellenberg (SP), S. González (SG), S. Gutiérrez (SU), S. Reyes (SR) y S. Zamudio (SZ).

\section{Pteridophyta}

\section{Adiantaceae}

Adiantum andicola Liebm. HD7387

Adiantum poiretii Wikstr. JM402, 801, 847; RG2495, 2542

Argyrochosma palmeri (Baken) Windham HD7181

Cheilanthes angustifolia Kunth JM725

Cheilanthes bonariensis (Willd.) Proctor HD7386; JM339; RG2546, 4010a, 4468

Cheilanthes hirsuta Link JM398, 420, 543, 724; JP2; RG2497, 2541, 2544, 2547b, 4481; SZ10143, 7193

Cheilanthes kaulfussii Kuntze HD7188; JM500, 843; RG2547a, 4000, 4476

Cheilanthes lendigera (Cav.) Sw. JM282; RG4001

Cheilanthes myriophylla Desv. JM684, 814; MC129; RG2545

Pellaea ovata (Desv.) Weath. JM345, 386, 520, 527

Pellaea ternifolia (Cav.) Link JM399, 1026; MC128

\section{Aspleniaceae}

Asplenium monanthes L. HD7191, 7392; JM286, 442, 800; RG2496, 2550

Dryopteris cinnamomea (Cav.) C.Chr. HD7385, 7189; MC130; RG2543

Dryopteris sp. JM572

Elaphoglossum muelleri (E.Fourn.) C.Chr. JM439, 806

Elaphoglossum petiolatum (Sw.) Urb. HD7185; JC1

Tectaria sp. JM747

Woodsia mollis (Kaulf.) J.Sm. HD7182; MC3

\section{Blechnaceae}

Woodwardia spinulosa M.Martens et Galeotti JM296, 757

\section{Dennstaedtiaceae}

Pteridium caudatum (L.) Maxon HD7391

Pteridium feei (W.Schaffn. ex Fée) Faull. JM327, 551; SUs/n

\section{Equisetaceae}

Equisetum myriochaetum Schltdl. et Cham. JM534, 941

Equisetum sp. HD7402

\section{Ophioglossaceae}

Botrychium virginianum (L.) Sw. JM445, 578

\section{Polypodiaceae}

Pecluma cupreolepis (A.M.Evans) M.G.Price JM432

Phlebodium araneosum (M.Martens et Galeotti) Mickel et Beitel HD7194, 7389; JM469

Phlebodium areolatum (Humb. et Bonpl. ex Willd.) J.Sm. RG4477

Pleopeltis mexicana (Fée) Mickel et Beitel JM325, 910

Pleopeltis polylepis (Roem. ex Kunze) T.Moore JM430

Pleopeltis peltata Scort. ex Alderw. var. peltata HD7183; JR425005; RG2548

Polypodium guttatum Maxon HD7187, 7390; JM320, 417, 431, 807; JR41992; MC4

Polypodium madrense J.Sm. HD7190

Polypodium polypodioides (L.) Watt. JM416

\section{Selaginellaceae}

Selaginella pallescens (C.Presl.) Spring HD7186; JC5; MC4, 6, 54 
Selaginella porphyrospora A.Braun JM450

Selaginella rupincola Underw. HD7196

\section{Gymnospermae}

\section{Pinaceae}

Pinus cembroides Zucc. JM285, 498; MC136

\section{Angiospermae \\ Dicotyledonae}

\section{Acanthaceae}

Berginia virgata Harv. ex Benth. et Hook.f. var. virgata SU3a

\section{Amaranthaceae}

Gomphrena serrata L. JM514; MC2, 47

Guilleminea densa (Willd. ex Roem. et Schult.) Moq. MC72, 126

\section{Anacardiaceae}

Schinus molle L. MC73

\section{Apiaceae}

Arracacia atropurpurea (Lehm.) Benth. et Hook.f. ex Hemsl. JM365, 443, 579, 939; JR42017

Arracacia tolucensis (Kunth) Hemsl. HD7160

Daucus montanus Humb. et Bonpl. ex Spreng. MCs/n

Donnellsmithia juncea (Humb. et Bonpl. ex Spreng.) Mathias et Constance SZ10799

Eryngium beecheyanum Hook.f. et Arn. MC53

Eryngium pectinatum C.Presl ex DC. RG2538

Eryngium serratum Cav. HD7163; JC1; JM353, 740; SZ10803, 10811

Hydrocotyle umbellata L. JM449

Rhodosciadium montanum (J.M.Coult. et Rose) Mathias et Constance JM474, 586, 732; MC61

\section{Apocynaceae}

Telosiphonia hypoleuca (Benth.) Henr. JC2, 91; JM1002; MC94

Vinca major L. MC160

\section{Aquifoliaceae}

Ilex dugesii Fernald JM436, 589, 950

\section{Asclepiadaceae}

Asclepias contrayerba Sessé et Moc. MC45

Asclepias linaria Cav. JM975; MC52

\section{Asteraceae}

Ageratina calaminthaefolia (Kunth) R.M.King et H.Rob. JM652; MC127

Ageratina glabrata (Kunth) R.M.King et H.Rob. JM253, 891, 893, 911, 913

Ageratina ligustrina (DC.) R.M.King et H.Rob. JM290, 765

Ageratina petiolaris (Moc. et Sessé ex DC.) R.M.King et H.Rob. JM896

Ageratina rhomboidea (Kunth) R.M.King et H. Rob. JM488

Ageratum corymbosum Zucc. JM346, 516, 660, 812, 848, 868, 874; JR42080; SZ10831

Alloispermum scabrum (Lag.) H.Rob. RG2452

Aphanostephus ramosissimus DC. JM262

Archibaccharis serratifolia (Kunth) S.F.Blake JM524, 734; JR42000, 42001, 42078; SZ10126, 10145

Artemisia absinthium L. JC8; MC157

Artemisia ludoviciana Nutt. ssp. mexicana (Willd. ex Spreng.) D.D.Keck JC4, s/n; MC120

Aster moranensis Kunth JM273; JR42002; MC98, 169 
Aster subulatus Michx. MC28

Baccharis heterophylla Kunth JD2582, JM920, 982; JR41996;

Baccharis serraefolia DC. JM772

Baccharis sulcata DC. JM357, 471, 699, 886; JR42081; 42082; MC142

Baccharis thesioides Kunth JR42013, 42014

Bahia schaffneri S.Watson MC59

Bidens angustissima Kunth JM611, 678; JR40262; MC1

Bidens aurea (Aiton) Sherff MC3, 97

Bidens ferulaefolia (Jacq.) DC. JM812a; MC88

Bidens odorata Cav. MC125, s/n; RG2494, 2502

Brickellia pedunculosa (DC.) Harc. et Beaman RG2466, 2533

Brickellia pendula (Schrad.) A.Gray JM703; JR42004

Brickellia sp. SZ10121

Carphochaete grahamii A.Gray JM499, 834, 845, 859; JR41995

Cirsium ehrenbergii Sch.Bip. EP2846

Conyza canadensis (L.) Cronquist var. glabrata (A.Gray) Cronquist RG2529

Conyza confusa Cronquist JR42079

Conyza filaginoides (DC.) Hieron JC2; MC5

Conyza gnaphalioides Kunth JM596, 674; MC10, 12

Conyza schiedeana (Less.) Cronquist JM352; JR42058

Coreopsis mutica DC. JM465

Cosmos bipinnatus Cav. MC2

Cosmos crithmifolius Kunth JM600; MC144

Dahlia coccinea Cav. JM419, 663, 1005; RG3982

Dyssodia tenuifolia (Cass.) Loes. JM865; MC39

Erigeron delphinifolius Willd. JM838; MC101

Erigeron scaberrimus (Less.) G.L.Nesom RZs/n

Eupatorium cardiophyllum B.L.Rob. MC159

Eupatorium deltoideum Jacq. JR42006; RG2485, 2537

Eupatorium glabratum Kunth JM877

Eupatorium pazcuarense Kunth JR42037

Eupatorium pycnocephalum Less. RG4489

Eupatorium sp. JM855

Galinsoga parviflora Cav. RG2460

Gamochaeta americana (Mill) Wedd. MC11

Gnaphalium oxyphyllum DC. RG2536

Gnaphalium purpurascens DC. JM526, 645; MC8

Grindelia inuloides Willd. JC12; RZs/n, SUs/n

Grindelia subdecurrens DC. MC78; RG2461, 2700

Grindelia sp. JR42012

Heterosperma pinnatum Cav. JM675

Heterotheca inuloides Cass. JM512; MC40

Hieracium abscissum Less. JM367, 651; MC20

Jaegeria hirta (Lag.) Less. JR42019, 42024; RG2519

Leucelene ericoides (Torr.) Greene JM97; MC67, 107, 108

Machaeranthera pinnatifida (Hook.) Shinners MC83

Melampodium perfoliatum (Cav.) Kunth JM511; MC1

Melampodium strigosum Stuessy JM391

Perymenium buphthalmoides DC. JM373; MC4

Perymenium mendezii DC. MC56

Pinaropappus roseus (Less.) Less. JM351, 974; MC81a, 85;

Piqueria triflora Hemsl. JR42032;

Piqueria trinervia Cav. JM264, 360, 515, 637, 733, 798, 818, 872, 884; RG2473; SU5

Psacalium megaphyllum (B.L.Rob. et Greenm.) Rydb. JM539, 611b, 698, 1045

Psacalium peltatum (Kunth) Cass. RG4470a

Psacalium radulifolium (Kunth) H.Rob. et Brettell EC5023 
Roldana angulifolia (DC.) H.Rob. et Brettell JM256, 288, 668, 710

Roldana hartwegii (Benth.) H.Rob. et Brettell JM636, 749

Roldana heracleifolia (Hemsl.) H.Rob. et Brettell JM387

Roldana sessilifolia (Hook. et Arn.) H.Rob. et Brettell JM571, 647, 691; RG2535, 3986, 4475

Sanvitalia ocymoides DC. MC74

Senecio peltiferus Hemsl. JP5

Senecio reticulatus DC. JR41993; RG2490

Senecio salignus DC. JM912

Senecio sinuatus Gilib. JR41999; SZ10125

Smallanthus maculatus (Cav.) H.Rob. JM390, 575

Sonchus oleraceus L. JM934; MC105

Stevia eupatoria (Spreng.) Willd. SUs/n

Stevia hirsuta DC. RG2469

Stevia jorullensis Kunth RG2539

Stevia latifolia Benth. RG2487

Stevia lucida Lag. JM272, 369, 547, 591; MC3; RG4491

Stevia monardaefolia Kunth JR42040

Stevia nepetifolia Kunth RG2474

Stevia ovata Willd. JM635, 646; RG2486, 4482

Stevia salicifolia Cav. JCs/n; JM411, 417a, 464, 531, 565

Stevia salicifolia Cav. var. salicifolia RG2476

Stevia serrata Cav. JM330, 397, 548, 631

Stevia viscida Kunth JC5

Tagetes lucida Cav. HD7138; JM311, 414, 567; MC3, 140; RG2463; SUs/n

Tagetes lunulata Ortega JM513; MCs/n

Tagetes micrantha Cav. JP5; MC2, 5, 27; SUs/n

Tagetes tenuifolia Cav. MC24

Tagetes sp. HD7151

Taraxacum officinale Weber ex F.H.Wigg. JM928

Vernonia steetzii Sch. Bip. MC120a

Viguiera buddleiaeformis (DC.) Benth. et Hook.f. ex Hemsl. JM690; MC47, 111

Viguiera linearis (Cav.) Sch. Bip. ex Hemsl. MC42

Viguiera sessilifolia DC. JC5, 7; JM425, 570, 653, 696, 713; MC3; RG4007

Zinnia angustifolia Kunth var. angustifolia JM705, 815; MC35

Zinnia bicolor (DC.) Hemsl. MC96

Zinnia peruviana (L.) L. MC5, 93, 119

\section{Begoniaceae}

Begonia gracilis Kunth JM301, 435, 505, 529, 563, 666; MC6; RG3997; SRG. 5

\section{Berberidaceae}

Berberis moranensis Schult. et Schult.f. JM263, 824, 901; SZ10836

\section{Betulaceae}

Alnus acuminata Kunth ssp. glabrata (Fernald) Furlow JM774

Alnus acuminata Kunth ssp. arguta (Schltdl.) Furlow MCs/n

\section{Bignoniaceae}

Tecoma stans (L.) Juss. ex Kunth MC46

\section{Brassicaceae}

Eruca sativa Mill. JM481

Erysimum capitatum (Douglas ex Hook.) Greene JM470, 730

Lepidium lasiocarpum Nutt. ex Torr. et A.Gray JM849; MC103

Lepidium virginicum L. RZ2

Rorippa nasturtium-aquaticum (L.) Hayek SUs/n 


\section{Buddlejaceae}

Buddleja cordata Kunth JM337, 808, 828

Buddleja sessiliflora Kunth JC1; JM 836, 873; MC168

\section{Cactaceae}

Echinocereus polyacanthus Engelm. JM495, 916, 956

Mammillaria densispina (J.M.Coult.) Orcutt JM493

Mammillaria petterssonii Hildm. JM497, 915

Mammillaria sp. (sect. Stylotele) JM493

Opuntia lasiacantha Pfeiff. JM494

Opuntia robusta J.C.Wendl. var. robusta JM491

\section{Caesalpiniaceae}

Bauhinia purpurea Wall. JSs/n

Senna multiglandulosa (Jacq.) H.S.Irwin et Barneby MC70; RS635

Senna atomaria L.f. RG2484

\section{Callitrichaceae}

Callitriche heterophylla Pursh. RG2504

\section{Campanulaceae}

Diastatea tenera (A.Gray) McVaugh JR42059

Lobelia laxiflora Kunth var. laxiflora JM616, 949; SZ8410

Lobelia fenestralis Cav. MC139

\section{Caprifoliaceae}

Lonicera pilosa (Kunth) Spreng. JC2; JM302, 426, 648, 820, 959; MC9; SZ10820, 11760

Sambucus nigra L. ssp. canadensis (L.) Bolli JC4

Symphoricarpos microphyllus Kunth RZs/n

\section{Caryophyllaceae}

Arenaria lanuginosa (Michx.) Rohrb. JM683,723; JR42027; RG2531

Cerastium nutans Raf. JM588, 751; JR41994

Cerastium sp. JR42033

Cerdia congestiflora Hemsl. MC50

Drymaria gracilis Schltdl. et Cham. RG2521

Drymaria laxiflora Benth. RG4469

Drymaria villosa Schltdl. et Cham. JM408, 670, 737

Drymaria xerophylla A.Gray JM671; MC32

Minuartia moehringioides (DC.) Mattf. HD7171; JM374, 535, 585, 714, 736, 753, 803; JR42020; RG4006; SZ10120, 10794

Paronychia mexicana Hemsl. JM641, 780, 870

Silene laciniata Cav. JM595, 692, 861; MC1, 90, 146, s/n; RG4483

\section{Chenopodiaceae}

Chenopodium ambrosioides L. MCs/n

Chenopodium graveolens Willd. JC3; JM388; RZs/n

\section{Cistaceae}

Helianthemum glomeratum ((Lag.) Lag. ex DC. JC1; JM261, 329, 661, 722, 768, 833, 863, 875, 962; JP3; MC4; RG2455; RZs/n

\section{Clusiaceae}

Hypericum galioides Lam. HD7125; JR42060; MC2

Hypericum philonotis Schltdl. et Cham. RG2500

Hypericum silenoides Juss HD7125 a; JM415, 536, 658, 726, 759, 826, 846; MC113 


\section{Convolvulaceae}

Convolvulus equitans Benth. MC44

Cuscuta mitriformis Engelm. JM712

Dichondra argentea Humb. et Bonpl. ex willd. JC4; JP3; MC23

Evolvulus alsinoides (L.) L. MC36

Evolvulus prostratus B.L.Rob. MC49

Evolvulus rotundifolius (S.Watson) Hallier f. MC37

Ipomoea capillacea (Kunth) G.Don HD7140; MC33

Ipomoea madrensis S.Watson EC5022

Ipomoea orizabensis (Pelletan) Ledeb. ex Steud. JP2, MC4, 29

\section{Cornaceae}

Cornus excelsa Kunth JC4; JM412, 604, 924, 933; RZs/n

\section{Crassulaceae}

Echeveria agavoides Lem. JM931

Echeveria mucronata Schltdl. JM404, 679

Echeveria secunda Booth ex Lindl. EC5018; HD7147; JM314, 347, 421, 957; SZ2550

Sedum fuscum Hemsl. JM819; MC19, 141

Sedum moranense Kunth EC5020; HD7139

Villadia misera (Lindl.) R.T.Clausen JM688, 707; MC79

\section{Cucurbitaceae}

Sicyos deppei G.Don MC1

\section{Ericaceae}

Arbutus arizonica (A.Gray) Sarg. SZ10136, 11346

Arbutus glandulosa M.Martens et Galeotti JM257, 813, 882, 895

Arbutus mollis Kunth SZ11356, 11357

Arbutus tessellata P.D.Sorensen HD7180; SZ10137, 11349

Arbutus xalapensis Kunth JM 778, 796

Arctostaphylos pungens Kunth JM259, 487, 822, 864, 876, 964; JR42062; RG2459

Comarostaphylis glaucescens (Kunth) Zucc. ex Klotzsch JM468, 593, 854, 921, 968; JR42065; SZ10134

Comarostaphylis polifolia (Kunth) Zucc. ex Klotzsch JM823

Pernettya ciliata (Schltdl. et Cham.) Small JM784, 817, 967

\section{Euphorbiaceae}

Acalypha sp. SZ10843

Euphorbia anychioides Boiss. JC1

Euphorbia dentata Michx. JM507

Euphorbia furcillata Kunth JM293, 428, 476, 756, 909, 961; JR41998; MC166

Euphorbia hirta L. var. procumbens (DC.) N.E.Br. JC3

Euphorbia macropus (Klotzsch et Garcke) Boiss. JM376, 987

Tragia nepetifolia Cav. var. nepetifolia JM946, MC3

\section{Fabaceae}

Astragalus ervoides Turcz. JM973; MC65

Cologania angustifolia Kunth JM364a, 478, 538

Cologania broussonetii (Balb.) DC. JM364, 630, 685, 715, 754, 985

Cologania obovata Schltdl. JM1025; MC26

Crotalaria rotundifolia (Walter) Walter ex J.F. Gmel. var. vulgaris Windler JM728; MC143

Dalea bicolor Humb. et Bonpl. ex. Willd. JM788a, 867

Dalea lutea Willd. JM598, 788; MC150

Dalea foliolosa (Aiton) Barneby var. citrina (Rydb.) Barneby JM470, 610; MC64

Desmodium aparines (Link) DC. JM614

Desmodium grahamii A.Gray JM382, 545, 654, 771 
Desmodium neomexicanum A.Gray JM569, 769

Erythrina leptorhiza Moc. et Sessé ex DC. JM930 Eysenhardtia platycarpa Pennell et Saff. MC147a Eysenhardtia polystachya (Ortega) Sarg. HD7152 Indigofera montana Rose MC138

Lathyrus parvifolius S.Watson JM681; MC16, 92

Lotus repens (G.Don) Sessé et Moc. ex Standl. et Steyerm. JM473, 609, 761; MC145

Macroptilium gibbosifolium (Ortega) A. Delgado MC104, s/n

Marina neglecta (B.L.Rob.) Barneby JM766

Medicago polymorpha L. JC6; JM832a; MC5, 60; RG2451

Phaseolus coccineus L. EC5025; JM532; SU2

Phaseolus maculatus Scheele JM371

Phaseolus pedicellatus Benth. JM452, 522, 767, 1015

Trifolium amabile Kunth JM349, 568, 720; MCs/n

Trifolium wormskioldii Lehm. var. ortegae (Greene) Barneby SUs/n

Vicia pulchella Kunth JM669

Zornia thymifolia Kunth JC4; JM362, 597, 995; MCs/n

\section{Fagaceae}

Quercus aristata Hook. et Arn. JM621

Quercus castanea Née JM312, 462, 463, 642, 731, 762, 763, 804

Quercus coccolobifolia Trel. JM284, 310, 317, 483, 558; SP12848

Quercus crassipes Humb. et Bonpl. JM489

Quercus eduardii Trel. JM622; MC134, 135; RG3992; SP12850

Quercus laeta Liebm. JM332, 619

Quercus laurina Bonpl. JM287, 306, 555, 556, 557, 592, 602, 603, 638, 906; SZ10133

Quercus microphylla Née JM960, 1006

Quercus obtusata Bonpl. JR42064; LM3252; MC137

Quercus potosina Trel. JM331, 378, 461, 508, 519, 559, 643

Quercus repanda Bonpl. JR42083

Quercus resinosa Liebm. JM300a, 620

Quercus rugosa Née EP2845; JM292, 305, 460, 605, 779; RA605; SZ10131

Quercus sideroxyla Bonpl. JM560, 633

\section{Garryaceae}

Garrya laurifolia Hartw. ex Benth. var. laurifolia HD7154; JM485, 892, 898, 907

Garrya laurifolia Hartw. ex Benth. var. macrophylla (Benth.) Dahling JM590

\section{Gentianaceae}

Gentiana spathacea Kunth MC143a

Gentianopsis detonsa (Rottb.) Ma ssp. lanceolata (Benth) J.M.Gill JM528, 613, 776

Halenia brevicornis (Kunth) G.Don JR42022; SU5b

\section{Geraniaceae}

Erodium cicutarium (L.) L'Hér. ex Aiton RZ2

Geranium bellum Rose HD7129; MC38

Geranium mexicanum Kunth JM304, 587

Geranium seemannii Peyr. JM 729; MC9, 99; RG2506

\section{Lamiaceae}

Agastache palmeri (B.L.Rob.) Standl. JM564

Cunila secunda S.Watson MC154

Lepechinia caulescens (Ortega) Epling JM363, 1036; MC1

Marrubium vulgare L. JM837, 971; MC75; RZs/n

Rosmarinus officinalis L. JC2; MC158

Salvia amarissima Ortega RG2475 
Salvia axilaris Moc. et Sessé HD7146; JM354, 383, 506

Salvia elegans Vahl JM252, 626, 708, 952; MC2; RG2483, 3967

Salvia greggii A.Gray JM748

Salvia lavanduloides Kunth JM662, 701

Salvia mexicana L. JM294, 380, 566; RG2481; SU5a

Salvia microphylla Kunth JCs/n

Salvia nana Kunth RG3990

Salvia patens Cav. JCs/n; JM307, 422, 727

Salvia polystachya Ortega RG2477

Salvia prunelloides Kunth JM303, 375, 459, 659, 989; MC34

Scutellaria coerulea Moc. et Sessé ex Benth. EC5021; JM381, 427, 479; MC5

Scutellaria sp. JM395, 686

Stachys agraria Schltdl. et Cham. JM389

Stachys coccinea Ortega JM440, 594; MC81, 84; RG3971

\section{Lentibulariaceae}

Pinguicula macrophylla Kunth HD7148; RG3979; SUs/n; SZ6368, 10813

Pinguicula moranensis Kunth JM309, 672

\section{Linaceae}

Linum schiedeanum Schltdl. et Cham. JM355; JR42023

\section{Loasaceae}

Mentzelia hispida Willd. MC55, 146a

Loranthaceae

Phoradendron forestierae B.L.Rob. et Greenm. JM276

\section{Lythraceae}

Heimia salicifolia Link MC95

\section{Malphigiaceae}

Aspicarpa hirtella Rich. MC37

Gaudichaudia cynanchoides Kunth MC145a, s/n

\section{Malvaceae}

Anoda cristata (L.) Schltdl. MC6, 115

Kearnemalvastrum subtriflorum (Lag.) D.M.Bates JC7; JR42049; RG2482

Modiola caroliniana (L.) G.Don JM350

Sida ciliaris L. MC117

Sphaeralcea angustifolia (Cav.) G.Don MC9; SR1

Urocarpidium limense (L.) Krapov. MC7; SU6

\section{Mimosaceae}

Acacia angustissima (Mill.) Kuntze MC149

Acacia berlandieri Benth. EV6672

Calliandra humilis Benth. MC66

Havardia elachystophylla A.Gray JM319, 466, 649; MC25

Mimosa aculeaticarpa Ortega JM983

\section{Nyctaginaceae}

Mirabilis jalapa L. JM384; MC6

Mirabilis melanotricha (Standl.) Spellenb. SZ10830

\section{Oleaceae}

Fraxinus uhdei (Wenz.) Lingelsh. JSs/n 


\section{Onagraceae}

Gaura hexandra Ortega MC57

Lopezia hirsuta Jacq. MC21

Lopezia racemosa Cav. MCs/n

Oenothera deserticola (Loes.) Munz JM760, 935

Oenothera pubescens Willd. ex Spring. JM395a, 396; MC102

Oenothera rosea L'Hér. ex Aiton JM927, 944; MC4, 112

Oenothera sp. JM517

\section{Orobanchaceae}

Conopholis alpina Liebm. JM372, 738, 917, 965; RG3963

\section{Oxalidaceae}

Oxalis corniculata L. JM359, 518, 839, 936, 980

Oxalis sp. HD7156

\section{Passifloraceae}

Passiflora exsudans Zucc. JM392, 697; MC5

\section{Phytolaccaceae}

Phytolacca icosandra L. JD2462; JM490; JP4; SUs/n

\section{Piperaceae}

Peperomia campylotropa A.W.Hill HD7137; JM438, 719

Peperomia galioides Kunth JM323, 455, 581

\section{Plantaginaceae}

Plantago australis Lam. JM409

Plantago australis Lam. ssp. hirtella (Kunth) Rahn JR42015

Plantago linearis Kunth var. mexicana (Link) Pilg. JM650, 739

Plantago linearis Kunth var. villosa Pilg. JM344, 789; RG3970

Plantago sp. RG2510

\section{Polemoniaceae}

Ipomopsis pinnata (Cav.) V.E.Grant MC94; SZ10842

Loeselia coerulea (Cav.) G.Don MC30

Loeselia mexicana (Lam.) Brand JC3; JM273a, 680, 795, 810, 869, 881; MC2, 132

\section{Polygalaceae}

Polygala alba Nutt. JM358

Polygala dolichocarpa S.F.Blake JM552

Polygala myrtilloides Willd. JM336, 429, 777

\section{Primulaceae}

Centunculus minimus L. HD7198

\section{Pyrolaceae}

Chimaphila umbellata (L.) W.P.C.Barton JM922; MC92

\section{Ranunculaceae}

Anemone mexicana Kunth HD7166; JD3976; JR42009; MC1; RG2508, 2513, 3984; SZ10805, 10130

Clematis dioica L. JM394; MC153

Delphinium pedatisectum Hemsl. MC58

Ranunculus hydrocharoides A.Gray JM340

Ranunculus petiolaris Kunth ex DC. JM447, 475, 573, 755; MC170

Thalictrum gibbosum Lechler MC77 


\section{Rhamnaceae}

Ceanothus coeruleus Lag. JC2; JM467

Rhamnus microphylla Humb. et Bonpl. ex Schult. JM486, 546, 969

\section{Rosaceae}

Alchemilla aphanoides Mutis ex L.f. JM504, 634

Alchemilla pringlei (Rydb.) Fedde JR42029; RG2509

Alchemilla procumbens Rose JM454, 830

Amelanchier denticulata (Kunth) K.Koch JM275, 338, 945

Cowania mexicana D.Don JM889

Crataegus mexicana Moc. et Sessé ex DC. JM254, 919, 954, 955a

Crataegus rosei Eggl. RG3980

Crataegus sp. JM958, LM3254

Prunus serotina Ehrh. ssp. capuli (Cav.) McVaugh JM900, 908; RZs/n

Rubus fagifolius Schltdl. et Cham. RG2523

Rubus pringlei Rydb. JM370, 926, 937

\section{Rubiaceae}

Bouvardia ternifolia (Cav.) Schltdl. JM368, 433, 618, 948

Bouvardia sp. JM979

Crusea diversifolia (Kunth) W.R.Anderson JM601; MC31

Crusea longiflora (Willd. ex Roem. et Schult.) W.R.Anderson JM1042; MC3, 121; SZ10828

Galium aschenbornii Nees et S.Schauer JM482, 687, 716, 811

Galium mexicanum Kunth GRs/n; JM743;

Galium sphagnophilum (Greenm.) Dempster HD7197

Galium uncinulatum DC. JM437, 584, 752; JR41997

Richardia gandarae Rzed. HD7141

Spermacoce verticillata L. MC63

\section{Rutaceae}

Casimiroa edulis Llave et Lex. JM841

\section{Salicaceae}

Salix bonplandiana Kunth JSs/n; MC171

\section{Sapindaceae}

Dodonaea viscosa Jacq. JM599, 866

\section{Saxifragaceae}

Heuchera orizabensis Hemsl. JM424, 925

Ribes affine Kunth JM984

\section{Scrophulariaceae}

Calceolaria tripartita Ruiz et Pav. RG2520

Castilleja arvensis Schltdl. et Cham. MC3, s/n

Castilleja canescens Benth. JM972

Castilleja lithospermoides Kunth RG2512

Castilleja tenuifolia M.Martens et Galeotti JM393, 562; MC148

Castilleja sp. JR42010

Lamourouxia multifida Kunth JM525, 561, 632

Lamourouxia rhinanthifolia Kunth EC5016; JM458; RG3999

Mecardonia procumbens (Mill.) Small JM406, 689; MC18; RG2525

Mimulus glabratus Kunth SUs/n

Penstemon campanulatus (Cav.) Willd. JM574, 617; RG2456, 3973

Penstemon imberbis (Kunth) Trautv. JM361, 456a, 530; MC17

Penstemon kunthii G.Don JM313, 324, 361, 577, 1021 
Penstemon roseus (Cerv. ex Sweet) G.Don EC5017; JD2583; MC2; RG2457; SZ10800

Penstemon schaffneri (Hemsl.) Straw JM326, 456, 750

Penstemon sp. SZ10122

Seymeria virgata (Kunth) Benth. JM477, 542, 608, 791; RG3960

Seymeria sp. JR42003

\section{Solanaceae}

Cestrum anagyris Dunal JM297, 379, 480, 576, 923, 966

Cestrum sp. HD7153

Datura stramonium L. JM630a; RZs/n

Jaltomata procumbens (Cav.) J.L.Gentry SU1

Lycianthes dejecta (Fernald) Bitter MC5

Physalis patula Mill. MC76

Physalis orizabae Dunal MC8

Physalis virginiana Mill. MC62

Solanum brachycarpum Correll JM673

Solanum cervantesii Lag. MCs/n; SZ10795

Solanum nigrescens M.Martens et Galeotti JM366, 405, 721; SUs/n

Solanum tuberosum L. JM444

\section{Tropaeolaceae}

Tropaeolum majus L. JC2; MCs/n; RZs/n

\section{Verbenaceae}

Glandularia bipinnatifida (Nutt.) Nutt. MC48

Priva mexicana (L.) Pers. MC2

Verbena carolina L. JM409; SZ10845

Verbena gracilis Desf. MC68

Verbena teucriifolia M.Martens et Galeotti JM831; MC48, s/n; RG2472; RZs/n

\section{Violaceae}

Viola aff. cochranei Ballard JM342, 827, 955

Viola guatemalensis W.Becker JM299, 744, 899

\section{Vitaceae}

Cissus sicyoides L. MC51

\section{Monocotyledonae}

Agavaceae

Polianthes geminiflora (Lex.) Rose MC14

Prochnyanthes mexicana (Zucc.) Rose MC80

\section{Alliaceae}

Allium kunthii G.Don JM550

Milla biflora Cav. JM700; MC11, 152

\section{Anthericaceae}

Echeandia flavescens (Schult. et Schult.f.) Cruden JM434, 523, 540, 717

Echeandia flexuosa Greenm. JM627

\section{Asphodelaceae}

Asphodelus fistulosus L. JM981; MC124; RG2489

\section{Bromeliaceae}

Tillandsia recurvata (L.) L. JM281, 448, 764, 858, 918, 978

Tillandsia sp. JM448 
Calochortaceae

Calochortus barbatus (Kunth) J.H.Painter HD7176; MC69

\section{Commelinaceae}

Commelina coelestis Willd. JM639, 667

Commelina dianthifolia Delile AR2492; HD7159

Commelina tuberosa L. RZ5; SU1a

Tradescantia crassifolia Cav. AR2494; HD7208; MC22

\section{Cyperaceae}

Bulbostylis juncoides (Vahl) Kük. ex Osten HD7142

Carex chordalis Liebm. JM951

Carex xalapensis Kunth JR42051

Carex sp. JM537

Cyperus hermaphroditus (Jacq.) Standl. JM341

Cyperus seslerioides Kunth JM553, 657

Cyperus sp. JR42026

Eleocharis sp. HD7170

Fimbristylis capillaris (L.) A.Gray JM322, 554, 656, 677, 718, 790, 862

\section{Iridaceae}

Sisyrinchium pringlei B.L.Rob. et Greenm. JM472, 533, 607, 785; MC87

\section{Juncaceae}

Juncus acuminatus Michx. MC1

Juncus effusus L. JM423, 940; MC1a

Juncus mexicanus Willd. ex Schult. et Schult.f. JM453, 903, 942

Juncus microcephalus Kunth MC119a

Juncus tenuis Willd. HD7175; JM835; MC1b, 119b

\section{Melianthaceae}

Zigadenus virescens (Kunth) J.F.Macbr. MC114; SZ10796

\section{Nolinaceae}

Dasylirion acrotrichum (Schiede) Zucc. JM902

\section{Orchidaceae}

Bletia reflexa Lindl. JM521; MC1, 151, s/n

Malaxis brachystachya (Rchb.f.) Kuntze JM709

Malaxis novogaliciana R.González ex McVaugh JJ488

\section{Poaceae}

Aegopogon cenchroides Humb. et Bonpl. ex Willd. HD7165; RG2458

Aegopogon tenellus (DC.) Trin. JM403, 544; MC118a

Aristida spanospicula Allred, Valdés-Reyna et Sánchez-Ken JM793

Aristida sp. JR42070

Brachypodium mexicanum (Roem. et Schult.) Link JM580; JR42046; MC147

Bromus anomalus Rupr. ex E.Fourn. JR42048

Bromus exaltatus Bernh. JM682, 1038

Bromus sp. JR42047

Cynodon dactylon (L.) Pers. JM623

Chascolytrum subaristatum (Lam.) Desv. JM446, 781

Eleusine indica (L.) Gaertn. JM624

Eragrostis intermedia Hitchc. JM328; JR42052

Eragrostis mexicana (Hornem.) Link JM509; MC123

Eragrostis trichodes (Nutt.) Alph.Wood JM549 
Eragrostis sp. JM549

Hilaria cenchroides Kunth JM625

Lycurus phleoides Kunth HD7124; JM676; JR42053

Muhlenbergia curvula Swallen JR42071

Muhlenbergia longiglumis Vasey JR42072

Muhlenbergia macrotis (Piper) Hitchc. RG4479

Muhlenbergia microsperma (DC.) Kunth JR42025

Muhlenbergia stricta (J.Presl) Kunth RG2515

Muhlenbergia versicolor Swallen JR42073

Muhlenbergia sp. JR42074, 42075

Nassella mucronata (Kunth) R.W.Pohl JM343

Panicum bulbosum Kunth MC110,156

Paspalum distichum L. JM629

Piptochaetium brevicalyx (E.Fourn.) Ricker JR42063

Piptochaetium fimbriatum (Kunth) Hitchc. HD7199

Piptochaetium virescens (Kunth) Parodi JM413, 510, 775; JR42045

Poa annua L. RG2522

Triniochloa stipoides (Kunth) Hitchc. JR42044

Rhynchelytrum repens (Willd.) C.E.Hubb. MC106

Schizachyrium sanguineum (Retz.) Alston JM792, 860

Xiridaceae

Xyris mexicana S.Watson MC13 